TRANSACTIONS OF THE

AMERICAN MATHEMATICAL SOCIETY

Volume 279, Number 2, October 1983

\title{
ON A QUESTION OF QUILLEN
}

BY

S. M. BHATWADEKAR AND R. A. RAO

\begin{abstract}
Let $R$ be a regular local ring, and $f$ a regular parameter of $R$. Quillen asked whether every projective $R_{f}$-module is free. We settle this question when $R$ is a regular local ring of an affine algebra over a field $k$. Further, if $R$ has infinite residue field, we show that projective modules over Laurent polynomial extensions of $R_{f}$ are also free.
\end{abstract}

Introduction. In [Q] Quillen posed the following

Question. Let $R$ be a regular local ring and $f$ a regular parameter of $R$. Are all finitely generated projective $R_{f}$-modules free?

An affirmative answer implies the

CONJECTURE (BASS-QUillen). Let $R$ be a regular local ring. Then every finitely generated projective $R[T]$-module is free.

Lindel [ $\mathbf{L}$, Theorem] has proved the Bass-Quillen conjecture when $R$ is the local ring of an affine algebra over a field $k$ at a regular point (not necessarily closed). However, it is not clear whether a positive solution to the Bass-Quillen conjecture implies the truth of Quillen's question. Therefore the latter is, apart from its application to the Bass-Quillen conjecture, of some independent interest. In this paper we settle the Quillen question affirmatively when $R$ is a regular local ring of an affine algebra over a field $k$.

Curiously, in this case we are able to reduce the Quillen question to the Bass-Quillen conjecture via the following interesting result (see Theorem 2.4).

THEOREM A. Let $R$ be any local ring. Then every stably free $R[T]$-module is free if and only if every stably free $R(T)$-module is free.

Swan has given an example of a four-dimensional regular affine complex algebra $A$ and a projective module $P$ over $A\left[Y, Y^{-1}\right]$ which is not extended from $A$ [Sw, §2]. Moreover he has shown that $P_{\mathfrak{p}}$ is free for every prime ideal $\mathfrak{p}$ of $A$. Theorem A shows the fact that $P_{\mathfrak{p}}$ is free is not accidental.

Swan's example leads us to consider projective modules over Laurent polynomial extensions of $R_{f}$. The constraints in the proof of Theorem 2.4 force us to reconstruct a different approach in this context. However in this approach we need to assume

Received by the editors July 12, 1982 and, in revised form, November 16, 1982.

1980 Mathematics Subject Classification. Primary 13C10; Secondary 13H05, 13B25. 
that $R$ has infinite residue field. In Theorem 3.2, we prove

THEOREM B. Let $R$ be a regular local ring of an affine algebra over a field $k$ with infinite residue field, and let $f$ be a regular parameter of $R$. Then every finitely generated projective $R_{f}\left[X_{1}, \ldots, X_{n}, Y_{1}^{ \pm 1}, \ldots, Y_{m}^{ \pm 1}\right]$-module is free.

Consequently, all finitely generated projective $R\left[X_{1}, \ldots, X_{n}, Y_{1}^{ \pm 1}, \ldots, Y_{m}^{ \pm 1}\right]-$ modules are free.

Mohan Kumar has answered the Quillen question affirmatively when $R$ is a power series ring over a field [Mo, Corollary 2]. In the last section of this paper we extend his arguments to show that every finitely generated projective $R_{f}\left[X_{1}, \ldots, X_{n}\right.$, $\left.Y_{1}^{ \pm 1}, \ldots, Y_{m}^{ \pm 1}\right]$-module is free. As an interesting application of this result we prove that every finitely generated projective $R(T)\left[X_{1}, \ldots, X_{n}, Y_{1}^{ \pm 1}, \ldots, Y_{m}^{ \pm 1}\right]$-module is free when $R$ is a power series ring over a field.

1. Preliminaries. Throughout this paper all rings will be commutative noetherian and all modules will be finitely generated.

(A) Patching technique. Let $\psi: B \rightarrow A$ be a homomorphism of rings and let $s$ be an element of $B$ such that:

(i) $s$ is a non-zero-divisor in $B$.

(ii) $\psi(s)$ is a non-zero-divisor in $A$.

(iii) $\psi$ induces an isomorphism $B / s B \simeq A / \psi(s) A$.

The commutative diagram

$$
\begin{array}{ccc}
B & \stackrel{\psi}{\rightarrow} & A \\
\downarrow & & \downarrow \\
B_{s} & \stackrel{\psi_{s}}{\rightarrow} & A_{s}
\end{array}
$$

resulting from a situation as above will be called a patching diagram.

We shall sometimes describe (1.1) as $B \stackrel{\psi}{\rightarrow} A$ is analytically isomorphic along $s$.

It is easy to see that diagram (1.1) is cartesian (i.e. $B$ is the fibre product of $B_{s}$ and $A$ over $A_{s}$ ).

Let $\mathbf{P}(R)$ denote the category of all finitely generated projective $R$-modules.

Given a patching diagram (1.1), the corresponding square

$$
\begin{array}{ccc}
\mathbf{P}(B) & \rightarrow & \mathbf{P}(A) \\
\downarrow & & \downarrow \\
\mathbf{P}\left(B_{s}\right) & \rightarrow & \mathbf{P}\left(A_{s}\right)
\end{array}
$$

is cartesian. This is a special case of a classical result of Milnor as shown in [Ry].

EXAMPles. (1) Covering diagrams. Let $s$ and $t$ be elements of a ring $B$ such that $B s+B t=B$. Assume $s$ is a non-zero-divisor in $B$. Then $B \rightarrow B_{t}$ is analytically isomorphic along $s$.

(2) Let $B=k\left[\left[Z_{1}, \ldots, Z_{p-1}\right]\right]\left[Z_{p}\right]$ and $A=k\left[\left[Z_{1}, \ldots, Z_{p}\right]\right]$, where $k$ is a field. Let $f$ be an element of $B$ which is a distinguished monic in $Z_{p}$, i.e. it is a monic polynomial 
in $Z_{p}$ with its lower degree coefficients belonging to the maximal ideal of $k\left[\left[Z_{1}, \ldots, Z_{p-1}\right]\right]$. As a consequence of the Weierstrass Preparation Theorem we see that

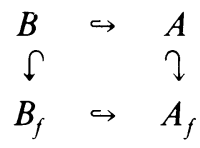

is a patching diagram.

(3) Let $(R, \mathfrak{m})$ be a local ring. A monic polynomial $f \in R[T]$ is called a Weierstrass polynomial if $f=T^{n}+a_{1} T^{n-1}+\cdots+a_{n}, a_{i} \in \mathrm{m}$ for $i=1,2, \ldots, n$.

Let $f \in R[T]$ be a Weierstrass polynomial. Then we have a patching diagram:

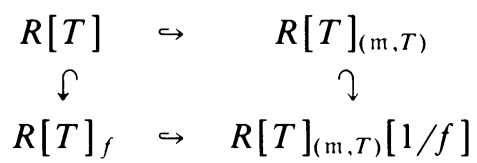

Proof. Undoubtedly, we do have an inclusion map $R[T] \hookrightarrow R[T]_{(\mathrm{m}, T)}$. Since $f$ is monic, $R[T] /(f(T))$ is semilocal, and any maximal ideal $\mathfrak{n}$ of it "sits" over $\mathrm{m}$. But since $f(T) \in \mathfrak{n}$ and $f$ is a Weierstrass polynomial, we have $T \in \mathfrak{n}$. Therefore $\mathfrak{n}=(\mathrm{m}, T)$. Thus $R[T] /(f(T))$ is local, and so

$$
R[T] /(f(T))=R[T] /(f(T))_{(\mathfrak{m}, T)}=R[T]_{(\mathfrak{m}, T)} /(f(T)) .
$$

(4) Let $\Lambda$ be a flat $\mathbf{Z}$-algebra. Then applying $\otimes_{\mathbf{Z}} \Lambda$ to the patching diagram (1.1), we get a new patching diagram

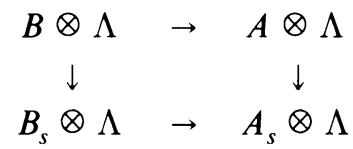

In applications here we shall take $\Lambda=\mathbf{Z}\left[X_{1}, \ldots, X_{n}, Y_{1}^{ \pm 1}, \ldots, Y_{m}^{ \pm 1}\right]$, a Laurent polynomial ring over $\mathbf{Z}$.

(B) Regular $k$-spots. Let $k$ be a field. By a regular spot over a field $k$ we mean a localisation $C_{\mathfrak{p}}$ of a finitely generated $k$-algebra $C$ at a regular prime $\mathfrak{p} \in \operatorname{Spec} C$.

Lindel [L, Proposition 2] analysed regular $k$-spots over perfect fields as étale extensions of rings of the type $K\left[Z_{1}, \ldots, Z_{n}\right]_{\left(\varphi\left(Z_{1}\right), Z_{2}, \ldots, Z_{n}\right)}$. We shall need the following finer analysis (see proof of [N, Theorem 2.8$]$ ):

Proposition. Let $(R, \mathrm{~m})$ be a regular $k$-spot over a perfect field $k$. Let $g \in \mathrm{m}$ and $f$ be any regular parameter of $R$ with $(g, f)$ a sequence. Then there exist a field $K \supset k$ and a regular $K$-spot $R^{\prime}$ such that:

(i) $R^{\prime}=K\left[Z_{1}, \ldots, Z_{d}\right]_{\left(\varphi\left(Z_{1}\right), \ldots, Z_{d}\right)}$, where $\varphi\left(Z_{1}\right) \in K\left[Z_{1}\right]$ is an irreducible monic. Moreover, we may assume $Z_{d}=f$.

(ii) $R^{\prime} \hookrightarrow R$ is an analytic isomorphism along $h$ for some $h \in g R \cap R^{\prime}$.

REMARK. If $R$ above has infinite residue field then the field $K$ is also infinite. 
2. The Quillen question for regular $k$-spots. We begin this section with a lemma.

LEMMA 2.1. Let $R$ be a semilocal ring and let $R[T]$ be a polynomial algebra in one variable over $R$. Let $J$ be an ideal of $R[T]$ containing a monic polynomial. Let $\mu\left(J / J^{2}\right)=d \geqslant 2$, where $\mu\left(J / J^{2}\right)$ denotes the minimal number of generators of $J / J^{2}$. Then there exist $g_{1}, \ldots, g_{d} \in J$ such that $\left(g_{1}, \ldots, g_{d}\right)=J$. Moreover, $g_{1}$ can be chosen to be monic.

Proof. Let $h_{1}, \ldots, h_{d}$ be elements of $J$ such that $\left(h_{1}, \ldots, h_{d}\right)+J^{2}=J$. Let $g \in J$ be a monic polynomial and let $g_{1}=h_{1}+g^{N}$. Then for $N \gg 0, g_{1}$ is monic. Moreover, $\mu\left(J / J^{2}+\left(g_{1}\right)\right)=d-1$. Let $R^{\prime}=R[T] /\left(g_{1}\right)$ and $J^{\prime}=J /\left(g_{1}\right)$. Then $J^{\prime}$ is an ideal of $R^{\prime}$ with $\mu\left(J^{\prime} / J^{\prime 2}\right)=d-1 \geqslant 1$. Since $g_{1}$ is monic, $R^{\prime}$ is semilocal. Therefore there exist $g_{2}^{\prime}, \ldots, g_{d}^{\prime} \in J^{\prime}$ such that $\left(g_{2}^{\prime}, \ldots, g_{d}^{\prime}\right)=J^{\prime}$. Let $g_{i}$ be a lift of $g_{i}^{\prime}$ in $J$ for $2 \leqslant i \leqslant d$. Then obviously $\left(g_{1}, g_{2}, \ldots, g_{d}\right)=J$.

In this paper $R(T)$ will denote the localisation of the polynomial algebra $R[T]$ by the multiplicatively closed subset of all monic polynomials in $R[T]$.

We use Lemma 2.1 in the proof of the following important theorem.

THEOREM 2.2. Let $R$ be a local ring and let $P$ be a projective $R(T)$-module such that $P \oplus R(T) \simeq R(T)^{d}$. Then there exists a projective $R[T]$-module $Q$ such that $Q \oplus$ $R[T] \simeq R[T]^{d}$ and $Q \otimes_{R[T]} R(T) \simeq P$.

Proof. Let $Y=T^{-1}$ and $\tilde{R}=R[Y]_{(\mathrm{m}, Y)}$ where $\mathrm{m}$ denotes the maximal ideal of $R$. Then $R[Y] \hookrightarrow \tilde{R}$ is analytically isomorphic along $Y$ and we have the patching diagram

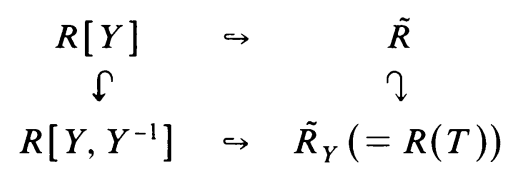

Let $\left[a_{1}, \ldots, a_{d}\right]$ denote a unimodular row of $R(T)^{d}$ defining the projective module $P$ over $R(T)$. Since $\tilde{R}_{Y}=R(T)$, without loss of generality we can assume that $a_{i} \in \tilde{R}$ for $1 \leqslant i \leqslant d$. Let $I$ be the ideal of $\tilde{R}$ generated by $a_{1}, \ldots, a_{d}$. If $\mu(I) \leqslant d-1$ then, since $\tilde{R}$ is local, one of the generators, say $a_{d}$, belongs to the ideal generated by the rest of the generators $a_{1}, \ldots, a_{d-1}$. But then $P \simeq R(T)^{d-1}$ and, taking $Q \simeq$ $R[T]^{d-1}$, we are through. Therefore we assume that $d=\mu(I)$.

Since $\left[a_{1}, \ldots, a_{d}\right]$ is a unimodular row over $\tilde{R}_{Y}$ we have $Y^{n} \in I$ for some positive integer $n$. Let $J=I \cap R[Y]$. Then, since $R[Y] \hookrightarrow \tilde{R}$ is analytically isomorphic along $Y$ and $Y^{n} \in I$, it follows that $J \tilde{R}=I$ and $\mu\left(J / J^{2}\right)=d \geqslant 2$. Therefore by Lemma $2.1, J=\left(g_{1}, \ldots, g_{d}\right)$ and $g_{1}$ is monic in $Y$. Since $\tilde{R}$ is local and $\mu(I)=d$, there exists an element $\sigma$ of $\mathrm{GL}_{d}(\tilde{R})$ such that $\left[g_{1}, \ldots, g_{d}\right] \sigma=\left[a_{1}, \ldots, a_{d}\right]$. Now consider the following short exact sequence:

$$
\begin{aligned}
0 \rightarrow R\left[Y, Y^{-1}\right] & \rightarrow R\left[Y, Y^{-1}\right]^{d} \rightarrow Q^{\prime} \rightarrow 0 \\
1 & \mapsto\left[g_{1}, \ldots, g_{d}\right]
\end{aligned}
$$


Since $Y^{n} \in J=\left(g_{1}, \ldots, g_{d}\right),\left[g_{1}, \ldots, g_{d}\right]$ is a unimodular row of $R\left[Y, Y^{-1}\right]^{d}$. Therefore $Q^{\prime}$ is a projective $R\left[Y, Y^{-1}\right]$-module and $P \simeq Q^{\prime} \otimes_{R\left[Y, Y^{-1}\right]} \tilde{R}_{Y}$. But $Q_{g_{1}}^{\prime}$ is free and $g_{1}$ is monic in $Y$. Hence by [Sw, Lemma 1.3] there exists a projective module $Q$ over $R\left[Y^{-1}\right]$ such that $Q^{\prime} \simeq Q \otimes_{R\left[Y^{-1}\right]} R\left[Y, Y^{-1}\right]$. Now $Q^{\prime} \oplus R\left[Y, Y^{-1}\right] \simeq R\left[Y, Y^{-1}\right]^{d}$ and therefore

$$
\left(Q \oplus R\left[Y^{-1}\right]\right)_{Y^{-1}} \simeq R\left[Y, Y^{-1}\right]^{d} .
$$

Hence, by [Q, Theorem 3 and Su, Theorem 1], $Q \oplus R\left[Y^{-1}\right] \simeq R\left[Y^{-1}\right]^{d}$, and we are through.

Corollary 2.3. Let $R$ be a two-dimensional local ring with $\frac{1}{2} \in R$. Then every stably free $R(T)$-module is free.

Proof. By Theorem 2.2 it suffices to show that every stably free $R[T]$-module is free. A proof of this can be found in [BR, 2.7]

As an easy consequence of Theorem 2.2 we get Theorem A.

THEOREM 2.4. Let $R$ be a local ring. Then every stably free $R[T]-$ module is free if and only if every stably free $R(T)$-module is free.

As an application of Theorem 2.4 we prove

THEOREM 2.5. Let $R$ be a regular spot of dimension $d$ over a field $k$, and let $f$ be a regular parameter of $R$. Then every finitely generated projective $R_{f}$-module is free.

Proof. Let $P$ be a projective $R_{f}$-module. If $d=1$ then $R_{f}$ is a field and there is nothing to prove. So we assume $d \geqslant 2$.

We first assume that $k$ is perfect.

Let $g$ be an element of $R$ such that $P_{g}$ is free. Without loss of generality we may assume that $g$ and $f$ have no common factors in $R$. Hence $(g, f)$ is a sequence in $R$.

Now by [N, Theorem 2.8], as stated in the preliminaries, there exist a field $K \supset k$ and a $K$-spot $R^{\prime}=K\left[Z_{1}, \ldots, Z_{d}\right]_{\left(\varphi\left(Z_{1}\right), \ldots, Z_{d}\right)}$ such that $R^{\prime} \leftrightarrow R$ is analytically isomorphic along $h$ for some $h \in g R \cap R^{\prime}$. Moreover $Z_{d}=f$.

Therefore $R_{Z_{d}}^{\prime} \hookrightarrow R_{f}$ is analytically isomorphic along $h$. Hence, since $P_{h}$ is free, by (1.1) there exists a projective $R_{Z_{d}}^{\prime}$-module $Q$ such that $P \simeq Q \otimes_{R_{Z_{d}}^{\prime}} R_{f}$. Therefore it is enough to prove that $Q$ is free.

Let $S=K\left[Z_{1}, \ldots, Z_{d-1}\right]_{\left(\varphi\left(Z_{1}\right), \ldots, Z_{d-1}\right)}$ and $T=Z_{d}^{-1}$. Then $R_{Z_{d}}^{\prime}=S(T)$. Now we are through in view of Theorem 2.4 and [L, Theorem].

In general we can reduce the problem to the case when the ground field $k$ is perfect as follows:

Let $k_{0}$ be the prime subfield of $k$. If $k$ is not perfect then $\operatorname{tr} \operatorname{deg}_{k_{0}} k \geqslant 1$. By the argument of Swan (see [L]) there exist a function field $k^{\prime}$ of $k_{0}$ contained in $k$ and a regular $k^{\prime}$-spot $R^{\prime}$ containing $f$ such that:

(1) $R^{\prime} \hookrightarrow R$;

(2) $f$ is a regular parameter of $R^{\prime}$;

(3) projective module $P$ extends from $R_{f}^{\prime}$;

(4) $\operatorname{tr} \operatorname{deg}_{k_{0}} k^{\prime} \geqslant 1$. 
Since $k^{\prime}$ is a function field of $k_{0}, R^{\prime}$ is a spot over $k_{0}$ also. Moreover, as $R^{\prime}$ contains $k^{\prime}$, by virtue of (4), $R^{\prime}$ has infinite residue field. This observation will be needed later in Theorem 3.2.

This completes the proof of Theorem 2.5.

3. Laurent polynomial extensions of $R_{f}$. We begin with a proposition which we shall use in the sequel.

Proposition 3.1. Let $B$ be a one-dimensional noetherian domain, and let $A$ be an overring of $B[T]$ which is contained in its quotient field. Assume that $A$ is a unique factorization domain. Then every projective $A\left[X_{1}, \ldots, X_{n}, Y_{1}^{ \pm 1}, \ldots, Y_{m}^{ \pm 1}\right]$-module is free.

Proof. We induct on $n+m$. If $n+m=0$, then by [R, Theorem 1.1(A)] every projective $A$-module is a direct sum of a free module and a rank one projective module. Since $A$ is a U.F.D., Pic $A=0$. Thus every projective $A$-module is free.

Assume $n+m>0$. Let $P$ be a projective $A\left[X_{1}, \ldots, X_{n}, Y_{1}^{ \pm 1}, \ldots, Y_{m}^{ \pm 1}\right]$-module.

Case (i). Let $n>0$. Let $S$ denote the multiplicatively closed subset of $A\left[X_{1}\right]$ consisting of all monic polynomials in $X_{1}$ with coefficients in $B$. Then $B\left[T, X_{1}\right]_{S}=$ $B\left(X_{1}\right)[T] \hookrightarrow A\left(X_{1}\right)$.

Therefore, by induction, $P \otimes A\left(X_{1}\right)\left[X_{2}, \ldots, X_{n}, Y_{1}^{ \pm 1}, \ldots, Y_{m}^{ \pm 1}\right]$ is free. Therefore, there exists a monic polynomial $f \in A\left[X_{1}\right]$ such that $P_{f}$ is free. By [Su, Theorem 1], $P$ is free.

Case (ii). Let $n=0$. Let $S^{\prime}$ denote the multiplicatively closed subset of $A\left[Y_{1}\right]$ consisting of monic polynomials in $Y_{1}$ with coefficients in $B$. Then $B\left[T, Y_{1}\right]_{S^{\prime}}=$ $B\left(Y_{1}\right)[T] \leftrightharpoons A\left(Y_{1}\right)$.

Therefore, by induction, $P \otimes A\left(Y_{1}\right)\left[Y_{2}^{ \pm 1}, \ldots, Y_{m}^{ \pm 1}\right]$ is free. By [Sw, Lemma 1.3], $P$ "extends" from $A\left[Y_{1}^{-1}, Y_{2}^{ \pm 1}, \ldots, Y_{m}^{ \pm 1}\right]$.

By case (i) above, $P$ is free.

THEOREM 3.2. Let $R$ be a regular spot of dimension $d$ over a field $k$ with infinite residue field. Let $f$ be a regular parameter of $R$. Then every finitely generated projective $R_{f}\left[X_{1}, \ldots, X_{n}, Y_{1}^{ \pm 1}, \ldots, Y_{m}^{ \pm 1}\right]$-module is free.

Proof. Let $P$ be a projective $R_{f}\left[X_{1}, \ldots, X_{n}, Y_{1}^{ \pm 1}, \ldots, Y_{m}^{ \pm 1}\right]$-module.

As in Theorem 2.5 we may assume that $k$ is perfect.

In this case we prove the result by induction on $d$. If $d \leqslant 2$ then $\operatorname{dim} R_{f} \leqslant 1$, and by [Sw, Corollary 1.4], $P$ is free. So let $d>2$. In general, by the corollary just mentioned, $P \otimes L\left[X_{1}, \ldots, X_{n}, Y_{1}^{ \pm 1}, \ldots, Y_{m}^{ \pm 1}\right]$ is free, where $L$ denotes the quotient field of $R$. Therefore, for some $g \in R, P_{g}$ is free. Without loss of generality we may assume that $g$ and $f$ have no common factors. Thus, $(g, f)$ is a sequence in $R$.

Now by [N, Theorem 2.8] as stated in the preliminaries, there exist an infinite field $K \supset k$ and a $K$-spot $R^{\prime}=K\left[Z_{1}, \ldots, Z_{d}\right]_{\left(\varphi\left(Z_{1}\right), Z_{2}, \ldots, Z_{d}\right)}$ such that $R^{\prime} \hookrightarrow R$ is analytically isomorphic along $h$ for some $h \in g R \cap R^{\prime}$. Moreover $Z_{d}=f$. Therefore $R_{Z_{d}}^{\prime} \rightarrow R_{f}$ is analytically isomorphic along $h$. Hence, as before, we may assume that $P$ extends from $R_{Z_{d}}^{\prime}\left[X_{1}, \ldots, X_{n}, Y_{1}^{ \pm 1}, \ldots, Y_{m}^{ \pm 1}\right]$. Call it $P$ still. Thus it suffices to prove that 
every projective module over $R_{Z_{d}}^{\prime}\left[X_{1}, \ldots, X_{n}, Y_{1}^{ \pm 1}, \ldots, Y_{m}^{ \pm 1}\right]$ is free. We note here that $d>2$. To prove this we shall use the following simple lemma (see, for example, [N, Proposition 1.11]).

LEMMA 3.3. Let $I$ be an ideal of a polynomial ring $B\left[T_{1}, \ldots, T_{n}\right]$ of height $\geqslant 2$. Then I contains a nonzero homogeneous polynomial.

Let $C$ denote $K\left[Z_{2}, \ldots, Z_{d}\right]$ and let $S$ denote the multiplicatively closed subset of $C$ consisting of all nonzero homogeneous polynomials in $C$. By Lemma 3.3, $\operatorname{dim} C_{S} \leqslant 1$.

Now $R_{Z_{d} S}^{\prime}$ is a localisation of $C_{S}\left[Z_{1}\right]$. By Proposition 3.1, $P_{S}$ is free. Hence for some $F \in S, P_{F}$ is free. We may assume that $Z_{d}$ does not divide $F$ in $C$.

Let $F=F_{1}+Z_{d} F_{2}, 0 \neq F_{1} \in K\left[Z_{2}, \ldots, Z_{d-1}\right]$.

Since $K$ is infinite, we may change $Z_{i}$ to $Z_{i}+\alpha_{i} Z_{2}$, for $3 \leqslant i \leqslant d-1$, for suitable $\alpha_{i} \in K$ and assume that $F(1,0, \ldots, 0) \neq 0$ with respect to the new set of variables, i.e. upto a unit $F$ is a monic in $Z_{2}$ with coefficients in $K\left[Z_{3}, \ldots, Z_{d}\right]$. Note that in view of the homogeneous change of variables, $F$ will be homogeneous with respect to the new set of variables.

Let

$$
\begin{aligned}
\tilde{R}^{\prime} & =K\left[Z_{1}, Z_{3}, \ldots, Z_{d}\right]_{\left(\varphi\left(Z_{1}\right), Z_{3}, \ldots, Z_{d}\right)}, \\
B & =\tilde{R}^{\prime}\left[X_{1}, \ldots, X_{n}, Y_{1}^{ \pm 1}, \ldots, Y_{m}^{ \pm 1}\right] \text { and } \\
A & =R^{\prime}\left[X_{1}, \ldots, X_{n}, Y_{1}^{ \pm 1}, \ldots, Y_{m}^{ \pm 1}\right] .
\end{aligned}
$$

By $\S 1(\mathrm{~A})$, Example 3, we have an analytic isomorphism $B\left[Z_{2}\right] \hookrightarrow A$ along $F$. Since $\left(F, Z_{d}\right)$ is a sequence in $B\left[Z_{2}\right]$ we get a patching diagram

$$
\begin{array}{ccc}
B_{Z_{d}}\left[Z_{2}\right] & \hookrightarrow & A_{Z_{d}} \\
\complement & & \urcorner \\
B_{Z_{d}}\left[Z_{2}\right]_{F} & \hookrightarrow & A_{Z_{d} F}
\end{array}
$$

Since $P_{F}$ is free, $P$ extends from $B_{Z_{d}}\left[Z_{2}\right]\left(=\tilde{R}_{Z_{d}}^{\prime}\left[Z_{2}, X_{1}, \ldots, X_{n}, Y_{1}^{ \pm 1}, \ldots, Y_{m}^{ \pm 1}\right]\right)$. By induction all projective $B_{Z_{d}}\left[Z_{2}\right]$-modules are free. Thus, $P$ is free.

This completes the proof of Theorem 3.2.

COROLlARY 3.4. Let $C$ be an affine algebra over a field $k$. Let $R=C_{\mathfrak{p}}$, where $\mathfrak{p}$ is a nonmaximal regular prime ideal of $R$. Let $f$ be a regular parameter of $R$. Then every finitely generated projective $R_{f}\left[X_{1}, \ldots, X_{n}, Y_{1}^{ \pm 1}, \ldots, Y_{m}^{ \pm 1}\right]$-module is free.

In the consequences below, $R$ will denote a regular spot over a field $k$ with infinite residue field.

CoROllaRY 3.5. All projective $R(T)\left[X_{1}, \ldots, X_{n}, Y_{1}^{ \pm 1}, \ldots, Y_{m}^{ \pm 1}\right]$-modules are free.

Proof. Since $R$ is local, $R(T)=R\left[T^{-1}\right]_{\left(\mathfrak{m}, T^{-1}\right)}\left[1 / T^{-1}\right]$. Thus, this is a particular case of Theorem 3.2 .

COROLlaRY 3.6. All projective $R\left[X_{1}, \ldots, X_{n}, Y_{1}^{ \pm 1}, \ldots, Y_{m}^{ \pm 1}\right]$-modules are free. 
Proof. Immediate from Corollary 3.5 by using Suslin's monic inversion theorem [Su, Theorem 1].

We have not been able to resolve whether projective $R_{f}\left[X_{1}, \ldots, X_{n}\right.$, $\left.Y_{1}^{ \pm 1}, \ldots, Y_{m}^{ \pm 1}\right]$-modules are free when $R$ has finite residue field. However, we believe it to be true, and towards this end we prove that all projective $R_{f}\left[X_{1}, \ldots, X_{n}, Y_{1}^{ \pm 1}, \ldots, Y_{m}^{ \pm 1}\right]$-modules of rank $\geqslant \operatorname{dim} R_{f}$ are free.

Before this:

Proposition 3.7. Let $B$ be a reduced noetherian ring of dimension $d$ and let $A$ be an overring of $B[X]$ which is contained in its total quotient ring. Then any stably free projective $A\left[X_{1}, \ldots, X_{n}, Y_{1}^{ \pm 1}, \ldots, Y_{m}^{ \pm 1}\right]$-module $P$ of rank $\geqslant d+1$ is free.

Proof. We induct on $n+m$. If $n+m=0$, this is a consequence of [R, Theorem 1.1(B)].

The general proof can be argued as in Proposition 3.1.

We now prove

THEOREM 3.8. Let $R$ be a regular spot of dimension $d$ over a field $k$ and $f$ be $a$ regular parameter of $R$. Then every projective $R_{f}\left[X_{1}, \ldots, X_{n}, Y_{1}^{ \pm 1}, \ldots, Y_{m}^{ \pm 1}\right]$-module of rank $\geqslant d-1$ is free. In particular if $d=3$, then every projective $R_{f}\left[X_{1}, \ldots, X_{n}, Y_{1}^{ \pm 1}, \ldots, Y_{m}^{ \pm 1}\right]$-module is free.

Proof. Since all projective modules over $R_{f}\left[X_{1}, \ldots, X_{n}, Y_{1}^{ \pm 1}, \ldots, Y_{m}^{ \pm 1}\right]$ are stably free, by Swan's theorem [Sw, Theorem 1.1] every projective $R_{f}\left[X_{1}, \ldots, X_{n}\right.$, $\left.Y_{1}^{ \pm 1}, \ldots, Y_{m}^{ \pm 1}\right]$-module of rank $\geqslant d$ is free. Our additional claim is that projective modules of rank $d-1$ are also free.

Via Swan's argument (see [L]) we may assume $k$ is perfect. As before, we may reduce the problem to the case when $R=K\left[Z_{1}, \ldots, Z_{d}\right]_{\left(\varphi\left(Z_{1}\right), Z_{2}, \ldots, Z_{d}\right)}$ for some field extension $K$ of $k$ and $f=Z_{d}$. Of course, $K$ may be finite now.

Let $P$ be a projective $R_{f}\left[X_{1}, \ldots, X_{n}, Y_{1}^{ \pm 1}, \ldots, Y_{m}^{ \pm 1}\right]$-module of rank $\geqslant d-1$. We prove that $P$ is free by induction on $d$. We know that if $d \leqslant 2$, then $P$ is free. Therefore we assume that $d>2$.

Let $\tilde{R}=K\left[Z_{1}, \ldots, Z_{d-1}\right]_{\left(\varphi\left(Z_{1}\right), Z_{2}, \ldots, Z_{d-1}\right)}$. Now $R_{Z_{d} Z_{d-1}}$ is a localisation of $\tilde{R}_{Z_{d-1}}\left[Z_{d}\right]$. Since $\operatorname{dim} \tilde{R}_{Z_{d-1}} \leqslant d-2$, by Proposition $3.7 P_{Z_{d-1}}$ is free.

Let $R^{\prime}=K\left[Z_{1}, \ldots, Z_{d-2}, Z_{d}\right]_{\left(\varphi\left(Z_{1}\right), Z_{2}, \ldots, Z_{d-2}, Z_{d}\right)}$. Then $R_{Z_{d}}^{\prime}\left[Z_{d-1}\right] \hookrightarrow R_{Z_{d}}$ is an analytic isomorphism along $Z_{d-1}$. Therefore, since $P_{Z_{d-1}}$ is free, by $\S 1(\mathrm{~A})$, Example 4, $P$ extends from $R_{Z_{d}}^{\prime}\left[Z_{d-1}, X_{1}, \ldots, X_{n}, Y_{1}^{ \pm 1}, \ldots, Y_{m}^{ \pm 1}\right]$. By induction, $P$ is free.

COROLLARY 3.9. Let $R$ be a regular $k$-spot of dimension $d$. Then:

(i) Every projective $R(T)\left[X_{1}, \ldots, X_{n}, Y_{1}^{ \pm 1}, \ldots, Y_{m}^{ \pm 1}\right]$-module of rank $\geqslant d$ is free.

(ii) Every projective $R\left[X_{1}, \ldots, X_{n}, Y_{1}^{ \pm 1}, \ldots, Y_{m}^{ \pm 1}\right]$-module of rank $\geqslant d$ is free.

We discuss some examples:

EXAMPLES. $\mathbf{R}$ will denote the field of real numbers.

(1) This example shows that Theorem 2.5 is not true for any arbitrary $f \in R$. Let $R=\mathbf{R}[X, Y, Z]_{(X, Y, Z)}, f=X^{2}+Y^{2}+Z^{2}$. It is easy to see that the projective module $P$ over $R_{f}$, given by the unimodular row $(X, Y, Z)$, is not free. 
(2) This example shows that Corollary 3.6 is not valid if we replace a regular $k$-spot by a nonsingular affine $k$-algebra. For another example see [Sw]. Let $R=\mathbf{R}[X, Y, Z] /\left(X^{2}+Y^{2}+Z^{2}-1\right)$ be the coordinate ring of the real 2-sphere $S^{2}$. Let $P$ be the projective $R\left[T, T^{-1}\right]$-module defined by the unimodular row $((1-x) T+(1+x), y, z)$, where $x, y, z$ denote the images in $R$ of $X, Y, Z$, respectively. Then $P$ is not extended from $R$. This is because $P /(T-1) P$ is free, whereas $P /(T+1) P$ is isomorphic to the tangent bundle of $S^{2}$ and so is nontrivial.

4. Laurent polynomial extensions of $k\left[\left[Z_{1}, \ldots, Z_{d}\right]\right]_{f}$ and $k\left[\left[Z_{1}, \ldots, Z_{d}\right]\right](T)$.

Proposition 4.1. Let $k$ be a field, and let $R=k\left[\left[Z_{1}, \ldots, Z_{d}\right]\right]$. Let $f \in R$ be a regular parameter of $R$. Then every finitely generated projective $R_{f}\left[X_{1}, \ldots, X_{n}\right.$, $\left.Y_{1}^{ \pm 1}, \ldots, Y_{m}^{ \pm 1}\right]$-module is free.

Proof. The case when $n=m=0$ was covered in [Mo]. Our proof covers this case too.

Since $R$ is complete and $f \in R$ is a regular parameter of $R$, we may, without any loss of generality, assume that $f=Z_{1}$.

We prove the result by induction on $d$. If $d=1, R_{Z_{1}}$ is a field and the result is due to Swan [Sw, Corollary 1.4]. Let $d \geqslant 2$. Let $P$ be a projective $R_{Z_{1}}\left[X_{1}, \ldots, X_{n}\right.$, $\left.Y_{1}^{ \pm 1}, \ldots, Y_{m}^{ \pm 1}\right]$-module. In general, by the above-mentioned corollary of Swan, there exists $g(\neq 0)$ in $R$ such that $P_{g}$ is free. We may assume that $Z_{1} \nmid g$.

Let $g=g_{1}+Z_{1} g_{2}, g_{1}(\neq 0)$ being a power series in $Z_{2}, \ldots, Z_{d}$.

After a change of variables involving $Z_{2}, \ldots, Z_{d}$ only, one may assume that $g_{1}$, and so $g$, is regular in $Z_{d}$. By the Weierstrass Preparation Theorem we can assume that $g$ is a Weierstrass polynomial in $k\left[\left[Z_{1}, \ldots, Z_{d-1}\right]\right]\left[Z_{d}\right]$ up to a unit.

Let $S=k\left[\left[Z_{1}, \ldots, Z_{d-1}\right]\right]$. Then, by $\S 1(\mathrm{~A})$, Examples 2 and 4 , we have an analytic isomorphism

$$
S\left[Z_{d}, X_{1}, \ldots, X_{n}, Y_{1}^{ \pm 1}, \ldots, Y_{m}^{ \pm 1}\right] \hookrightarrow R\left[X_{1}, \ldots, X_{n}, Y_{1}^{ \pm 1}, \ldots, Y_{m}^{ \pm 1}\right]
$$

along $g$. Since $\left(g, Z_{1}\right)$ is a sequence we have an analytic isomorphism

$$
S_{Z_{1}}\left[Z_{d}, X_{1}, \ldots, X_{n}, Y_{1}^{\prime \prime}, \ldots, Y_{m}^{\prime \prime}\right] \hookrightarrow R_{Z_{1}}\left[X_{1}, \ldots, X_{n}, Y_{1}^{ \pm 1}, \ldots, Y_{m}^{ \pm 1}\right]
$$

along $g$.

Since $P_{g}$ is free, $P$ extends from $S_{Z_{1}}\left[Z_{d}, X_{1}, \ldots, X_{n}, Y_{1}^{ \pm 1}, \ldots, Y_{m}^{ \pm 1}\right]$. Induction prevails.

Now we prove the main result of this section, which is the analogue of Corollary 3.5 when $R$ is complete.

THEOREM 4.2. Let $k$ be a field. Then every finitely generated projective $k\left[\left[Z_{1}, \ldots, Z_{d}\right]\right](T)\left[X_{1}, \ldots, X_{n}, Y_{1}^{ \pm 1}, \ldots, Y_{m}^{ \pm 1}\right]$-module is free.

Proof. Let $R=k\left[\left[Z_{1}, \ldots, Z_{d}\right]\right], A=R\left[T^{-1}\right]_{\left(Z_{1}, \ldots, Z_{d}, T^{-1}\right)}$. It is easy to see that the natural inclusion map $A \hookrightarrow R\left[\left[T^{-1}\right]\right]$ is analytically isomorphic along $T^{-1}$.

Observe that since $R$ is local, $A_{T^{-1}}=R(T)$. 
By $§ 1(A)$, Example 4, we have a patching diagram

$$
\begin{aligned}
& A\left[X_{1}, \ldots, X_{n}, Y_{1}^{ \pm 1}, \ldots, Y_{m}^{ \pm 1}\right] \quad \leftrightarrow \quad R\left[\left[T^{-1}\right]\right]\left[X_{1}, \ldots, X_{n}, Y_{1}^{ \pm 1}, \ldots, Y_{m}^{ \pm 1}\right] \\
& R(T)\left[X_{1}, \ldots, X_{n}, Y_{1}^{ \pm 1}, \ldots, Y_{m}^{ \pm 1}\right] \quad \hookrightarrow \quad R\left[\left[T^{-1}\right]\right]_{T^{-1}}\left[X_{1}, \ldots, X_{n}, Y_{1}^{ \pm 1}, \ldots, Y_{m}^{ \pm 1}\right]
\end{aligned}
$$

Since by Proposition 4.1, all projective modules over $R\left[\left[T^{-1}\right]\right]_{T^{-1}}\left[X_{1}, \ldots, X_{n}\right.$, $\left.Y_{1}^{ \pm 1}, \ldots, Y_{m}^{ \pm 1}\right]$ are free, $P$ extends from $A\left[X_{1}, \ldots, X_{n}, Y_{1}^{ \pm 1}, \ldots, Y_{m}^{ \pm 1}\right]$.

Therefore, it suffices to prove that every projective $A\left[X_{1}, \ldots, X_{n}, Y_{1}^{ \pm 1}, \ldots, Y_{m}^{ \pm 1}\right]$ module is free.

We can find a $g \in R$ such that $P_{g}$ is free. We may, without loss of generality, assume that $g$ is a Weierstrass polynomial in $Z_{d}$ with coefficients in $S=$ $k\left[\left[Z_{1}, \ldots, Z_{d-1}\right]\right]$. Then $S\left[Z_{d}\right] \hookrightarrow R$ is an analytic isomorphism along $g$. Consequently $B\left[Z_{d}\right] \hookrightarrow A$ is an analytic isomorphism along $g$ as by $\S 1(\mathrm{~A})$, Examples 2 and 3 , where $B=S\left[T^{-1}\right]_{\left(Z_{1}, \ldots, Z_{d-1}, T^{-1}\right)}$.

By $§ 1(A)$, Example 4, we have a patching diagram

$$
\begin{aligned}
B\left[Z_{d}, X_{1}, \ldots, X_{n}, Y_{1}^{ \pm 1}, \ldots, Y_{m}^{ \pm 1}\right] & \hookrightarrow A\left[X_{1}, \ldots, X_{n}, Y_{1}^{ \pm 1}, \ldots, Y_{m}^{ \pm 1}\right] \\
\downarrow & \downarrow \\
B\left[Z_{d}\right]_{g}\left[X_{1}, \ldots, X_{n}, Y_{1}^{ \pm 1}, \ldots, Y_{m}^{ \pm 1}\right] & \rightarrow A_{g}\left[X_{1}, \ldots, X_{n}, Y_{1}^{ \pm 1}, \ldots, Y_{m}^{ \pm 1}\right]
\end{aligned}
$$

Patch $P$ on $A\left[X_{1}, \ldots, X_{n}, Y_{1}^{ \pm 1}, \ldots, Y_{m}^{ \pm 1}\right]$ and a suitable free module $F$ on $B\left[Z_{d}\right]_{g}\left[X_{1}, \ldots, X_{n}, Y_{1}^{ \pm 1}, \ldots, Y_{m}^{ \pm 1}\right]$ via an isomorphism over $A_{g}\left[X_{1}, \ldots, X_{n}\right.$, $\left.Y_{1}^{ \pm 1}, \ldots, Y_{m}^{ \pm 1}\right]$ to get a projective module $P^{*}$ over $B\left[Z_{d}, X_{1}, \ldots, X_{n}, Y_{1}^{ \pm 1}, \ldots, Y_{m}^{ \pm 1}\right]$ such that

$$
P \simeq P^{*} \otimes A\left[X_{1}, \ldots, X_{n}, Y_{1}^{ \pm 1}, \ldots, Y_{m}^{ \pm 1}\right] .
$$

Since $P_{g}^{*} \simeq F$, by [Su, Theorem 1], $P^{*}$ is free. Thus, $P$ is free.

NOTE ADDED IN PROOF. Later, the second author has extended Theorem B in a preprint titled On Projective $R_{f_{1} \cdots f_{t}}$-Modules.

\section{REFERENCES}

[BR] S. M. Bhatwadekar and A. Roy, Inversion of monic polynomials and existence of unimodular elements (preprint).

[L] H. Lindel, On a question of Bass, Quillen and Suslin concerning projective modules over polynomial rings, Invent. Math. 65 (1981), 319-323.

[Mo] N. Mohan Kumar, On a question of Bass-Quillen, J. Indian Math. Soc. 43 (1979), 13-18.

[N] B. S. Nashier, Efficient generation of ideals in polynomial rings (preprint).

[Q] D. Quillen, Projective modules over polynomial rings, Invent. Math. 36 (1976), 167-171.

[R] R. A. Rao, Stability theorems for overrings of polynomial rings, II, J. Algebra 78 (1982), 337-444.

[Ry] A. Roy, Application of patching diagrams to some questions about projective modules, J. Pure Appl. Algebra 24 (1982), 313-319.

[Su] A. A. Suslin, Projective modules over a polynomial ring are free, Soviet Math. Dokl. 17 (1976), 1160-1164 (English translation).

[Sw] R. G. Swan, Projective modules over Laurent polynomial rings, Trans. Amer. Math. Soc. 237 (1978), $111-120$.

School of Mathematics, Tata Institute of Fundamental ReSEarch, Bombay 400 005, India 